\title{
SOME SUFFICIENT CONDITIONS FOR QUINTIC RESIDUACITY
}

\author{
YUN-CHENG ZEE
}

\begin{abstract}
It is shown that for a prime $p$ of the form $5 f+1$, a prime $q>5$ is a quintic residue $(\bmod p)$ if $u \equiv 0, v \equiv k w$ or $u \equiv k w, v \equiv 0$ $(\bmod q)$, where $k$ satisfies $k^{2} \equiv-3,5$ or $-15(\bmod q)$.
\end{abstract}

In his study of cyclotomy of order 5, L. E. Dickson [1, Theorem 8] showed that for each prime $\equiv 1(\bmod 5)$, there are exactly four simultaneous solutions of the Diophantine equations

$$
\begin{gathered}
16 p=x^{2}+50 u^{2}+50 v^{2}+125 w^{2}, \\
x w=v^{2}-u^{2}-4 u v,
\end{gathered}
$$

with $x \equiv 1(\bmod 5)$. If $(x, u, v, w)$ is one solution, the other three are $(x,-u$, $-v, w),(x, v,-u, w)$ and $(x,-v, u,-w)$. The values of $x, u, v$ and $w$ have been used in giving criteria for the quintic residuacity of the primes $q=2,3$ [2, pp. 13, 15], $5[4$, p. 122]. E. Lehmer [4, p. 124] showed that $q$ is a quintic residue $(\bmod p)$ if $u \equiv v \equiv w \equiv 0(\bmod q)$. A more general result giving a sufficient condition for the $r$ th power residuacity of $q$ is due to J. B. Muskat [6, Theorem 3]. When $r=5$, Muskat's condition, restated in terms of $x, u, v$ and $w$, becomes $u \equiv v \equiv 0(\bmod q)$.

Let $p$ be a prime of the form ef $+1, g$ a primitive root of $p$ and $\xi$ a $p$ th root of unity. The periods $\eta_{k}$, where $k=0,1, \ldots, e-1$, are defined by

$$
\eta_{k}=\sum_{t=1}^{f-1} \xi^{g^{e t+k}} .
$$

The equation

$$
\varphi(y)=\prod_{i=0}^{e-1}\left(y-\eta_{i}\right)=0
$$

is called the period equation of degree $e$. A theorem of Kummer [3, p. 436] states that if $e$ is a prime, then each prime divisor of the numbers represented by $\varphi(y)$ is an $e$ th power residue $(\bmod p)$. The reduced period equation

$$
F(z)=\prod_{i=1}^{e-1}\left(z-\rho_{i}\right)=0
$$

with the roots $\rho_{i}=e \eta_{i}+1$ is simpler than $\varphi(y)=0 . F(z)$ and $\varphi(y)$ are

Received by the editors December 11, 1974 and, in revised form, March 17, 1975.

AMS (MOS) subject classifications (1970). Primary 10A15; Secondary 10C05, 12C20.

Key words and phrases. Cyclotomy, cyclotomic numbers, quintic residuacity, period, period equation. 
related by $e^{e} \varphi(y)=F(z)$, where $z=e y+1$. The following lemma is then obvious:

LEMMA. If $e$ is a prime, then each prime divisor $\neq e$ of the numbers represented by $F(z)$ is an eth power residue $(\bmod p)$.

THEOREM. Let $p$ be a prime of the form $5 f+1$. A prime $q>5$ is a quintic residue $(\bmod p)$ if $u \equiv 0, v \equiv k w$ or $u \equiv k w, v \equiv 0(\bmod q)$, where $k$ satisfies $k^{2} \equiv-3,5$ or $-15(\bmod q)$.

Proof. The reduced period equation of degree 5 is $[2,(10)]$

$$
\begin{aligned}
F(z)= & z^{5}-10 p z^{3}-5 p x z^{2}-5 p\left[\left(x^{2}-125 w^{2}\right) / 4-p\right] z \\
& +p^{2} x-p\left[x^{3}+625\left(u^{2}-v^{2}\right) w\right] / 8 .
\end{aligned}
$$

For simplicity, congruences will be modulo $q$ throughout. Assume $u \equiv 0$, $v \equiv k w \not z 0$. Let $j$ satisfy $j k \equiv 1$. By (2), $x w \equiv k^{2} w^{2}$, so that $w \equiv j^{2} x$ and $v \equiv j x$. Substituting $u, v$ and $w$ into (1) and (3) yields

$$
\begin{gathered}
16 p \equiv\left(125 j^{4}+50 j^{2}+1\right) x^{2}, \\
8 F(z) \equiv 8 z^{5}-80 p z^{3}-40 p x z^{2}-5 p\left[2 x^{2}\left(1-125 j^{4}\right)-8 p\right] z \\
+8 p^{2} x-p x^{3}\left(1-625 j^{4}\right),
\end{gathered}
$$

respectively. In (5), let $z=x$ and simplify:

$$
8 F(x) \equiv x\left[8 x^{4}+\left(1875 j^{4}-131\right) p x^{2}+48 p^{2}\right] .
$$

Multiplying by 16 and applying (4) give

$$
\begin{array}{rl}
128 & F(x) \equiv x^{5}\left[128+\left(1875 j^{4}-131\right)\left(125 j+50 j^{2}+1\right)\right. \\
& \left.+3\left(125 j^{4}+50 j^{2}+1\right)^{2}\right] \\
& \equiv x^{5}\left[128+\left(125 j^{4}+50 j^{2}+1\right)\left(2250 j^{4}+150 j^{2}-128\right)\right] \\
& \equiv 6250 x^{5} j^{2}\left(45 j^{6}+21 j^{4}-j^{2}-1\right) \\
& \equiv 6250 x^{5} j^{2}\left(3 j^{2}+1\right)^{2}\left(5 j^{2}-1\right) .
\end{array}
$$

Hence

$$
2^{6} k^{8} F(x) \equiv(5 x)^{5}\left(3+k^{2}\right)^{2}\left(5-k^{2}\right) .
$$

Since $q \neq 2$, the last congruence implies that if $k^{2} \equiv-3$ or 5 , then $F(x) \equiv 0$ or $q \mid F(x)$. By the Lemma, $q$ is a quintic residue, $(\bmod p)$. Now, let $z=0$ in (5) and simplify:

$$
8 F(0) \equiv p x\left[8 p-x^{2}\left(1-625 j^{4}\right)\right] .
$$

Multiply by 2 and apply (4):

$$
\begin{aligned}
16 F(0) & \equiv p x^{3}\left[\left(125 j^{4}+50 j^{2}+1\right)-\left(1-625 j^{4}\right)\right] \\
& \equiv 50 p x^{3} j^{2}\left(15 j^{2}+1\right) .
\end{aligned}
$$

Hence 


$$
2^{3} k^{4} F(0) \equiv 5^{2} p x^{3}\left(15+k^{2}\right)
$$

By the Lemma if $k^{2} \equiv-15, q$ is a quintic residue $(\bmod p)$. If we assume $u \equiv k w \not \geq 0, v \equiv 0$ and let $j k \equiv 1$, we get $w \equiv-j^{2} x, u \equiv-j x$. Substitutions of $u, v$ and $w$ into (1) and (3) yield again (4) and (5) respectively, thus leading to the same condition on $k$. This completes the proof.

It is noted that for $q=7$, the sufficient condition in the last theorem becomes $u \equiv 0, v \equiv \pm 2 w$ or $u \equiv \pm 2 w, v \equiv 0$, which is a partial restatement of Muskat's condition (see [5, Theorem 2]).

We give an illustration for $q=11$. Since -3 and -15 are quadratic nonresidues $(\bmod 11)$, the condition on $k$ is reduced to $k^{2} \equiv 5(\bmod 11)$. For primes of the form $5 f+1$ less than 2,000 this condition yields five primes, of which 11 is a quintic residue, as given by the following table:

\begin{tabular}{r:rrr:r:r:r:r|r}
$p$ & $x$ & $u$ & $v$ & $w$ & $k$ & ind $11(\bmod p)$ \\
\hline 311 & -49 & 7 & 0 & 1 & 7 & 135 & 1 \\
661 & 1 & 0 & -3 & 9 & -4 & 380 & 1 \\
691 & 41 & -2 & 11 & 5 & 4 & 335 & । \\
751 & 71 & 4 & 11 & -1 & -4 & 715 & । \\
1181 & -64 & 0 & 16 & -4 & -4 & 160 & 1 \\
\hline
\end{tabular}

The author wishes to thank Professor Muskat for the use of his collection of data on the cyclotomic numbers [1] of order 5 from which the values of $x$, $u, v$ and $w$ were computed at the Computer Center of the California State University, Fullerton. The author is grateful to the referee for his valuable suggestions.

\section{REFERENCES}

1. L. E. Dickson, Cyclotomy, higher congruences and Waring's problem, Amer. J. Math. 57 (1935), 391-424.

2. E. Lehmer, The quintic character of 2 and 3, Duke Math. J. 18 (1951), 11-18. 7 12, 6779

3. Period equations applied to difference sets, Proc. Amer. Math. Soc. 6 (1955), 433-442. MR 16, 904.

4. __ Artiads characterized, J. Math. Anal. Appl. 15 (1966), 118-131. MR 34 \# 1261.

5. _ On the divisors of the discriminant of the period equation, Amer. J. Math. 90 (1968), 375-379. MR 37 \#2718.

6. J. B. Muskat, Reciprocity and Jacobi sums, Pacific J. Math. 20 (1967), 275-280. MR 35 \#1543.

Department of Mathematics, California State University, Fullerton, California 92634 